\title{
Inverted Type-I CdS/CdSe Core/Crown Colloidal Quantum Ring
}

\author{
Sumanta Bose, ${ }^{1}$ Savas Delikanli, ${ }^{1,2}$ Mehmet Zafer Akgul, ${ }^{2}$ Yuan Gao, ${ }^{1}$ \\ Weijun Fan, ${ }^{1, *}$ Dao Hua Zhang, ${ }^{1,3 \dagger}$ Hilmi Volkan Demir ${ }^{1,2,3}$ \\ ${ }^{l}$ School of Electrical and Electronic Engineering, Nanyang Technological University, Singapore 639798 \\ ${ }^{2}$ Department of Physics, Department of Electrical and Electronics Engineering and UNAM, Bilkent University, Ankara, Turkey 06800 \\ ${ }^{3}$ School of Physical and Mathematical Sciences, Nanyang Technological University, Singapore 639798 \\ E-mail address: ${ }^{*}$ ewjfan@ntu.edu.sg, ${ }^{\dagger}$ edhzhang@ntu.edu.sg, ${ }^{\ddagger}$ hvdemir@ntu.edu.sg
}

Inverted Type-I quantum rings (QRs) are a recently developed class of nanostructure in which a lower bandgap material is laterally grown as a crown of same thickness around a higher bandgap nanoplatelet (NPL) core [1,2], e.g. core/crown $\mathrm{CdS} / \mathrm{CdSe}$ as shown in Fig. 1(a). For this work, we have colloidally synthesized 3 monolayer (ML) thick CdS/CdSe QR samples using seed-mediated method, by using 3ML CdS NPLs as the core seed [3]. Subsequently, continuous injection of Se precursor, elemental Se dissolved in octadecene (ODE), $\mathrm{Cd}$ precursor and $\mathrm{Cd}\left(\mathrm{CH}_{3} \mathrm{CO}_{2}\right)_{2}$ led to the growth of CdSe crown. The ring width was controlled by tuning the injection amount to regulate the extent of the lateral size of the crown coating. A TEM image of the $3 \mathrm{ML}$ CdS/CdSe QRs is shown in Fig. 1(b), which suggests atomically flat lateral growth of CdSe on CdS NPLs, as it has larger average lateral size $(30 \pm 10 \mathrm{~nm})$ compared to the CdS core only NPLs. We have measured the photoluminescence (PL) and absorption spectra of CdS/CdSe QRs at different phases of crown growth. Fig. 1(c) shows the PL and absorption spectra for two contrasting cases having a (i) thin, and a (ii) thick CdSe crown. As the crown thickness increases, there is lesser extent of lateral confinement, and thus a red-shift in the PL peak position. Also the linewidth of the emission spectra narrows down as more CdSe is deposited in the crown. This is owing to the randomly dispersed CdSe islands of varying sizes formed around the CdS NPLs at the initial phase of the CdSe crown growth resulting in a normal distribution of energy levels. For a sufficiently thick CdSe crown, the electronic structure and optical properties of CdS/CdSe QR is identical to CdSe NPLs alone, characterized by narrower linewidths. Moreover, we have verified that the emission peak of the $3 \mathrm{ML} \mathrm{CdS/CdSe}$ QRs is spectrally tunable between the peak emissions of 3ML CdS NPL core only (382 nm) and $3 \mathrm{ML}$ CdSe NPL core only $(462 \mathrm{~nm})$. In terms of the absorption spectra, as we begin to grow the CdSe crown, there is a gradual emergence of the primary and secondary CdSe absorption peaks at $460 \mathrm{~nm}$ (electron-heavy hole) and $431 \mathrm{~nm}$ (electron-light hole) respectively, as can be seen in Fig. 1(c-ii). On the other hand, for thin CdSe crowns, these peaks are undeterminable, while the overlapping primary and secondary absorption peaks of CdS at 382 $\mathrm{nm}$ (electron-heavy hole) and $373 \mathrm{~nm}$ (electron-light hole) are more prominent, as can be seen in Fig. 1(c-i).
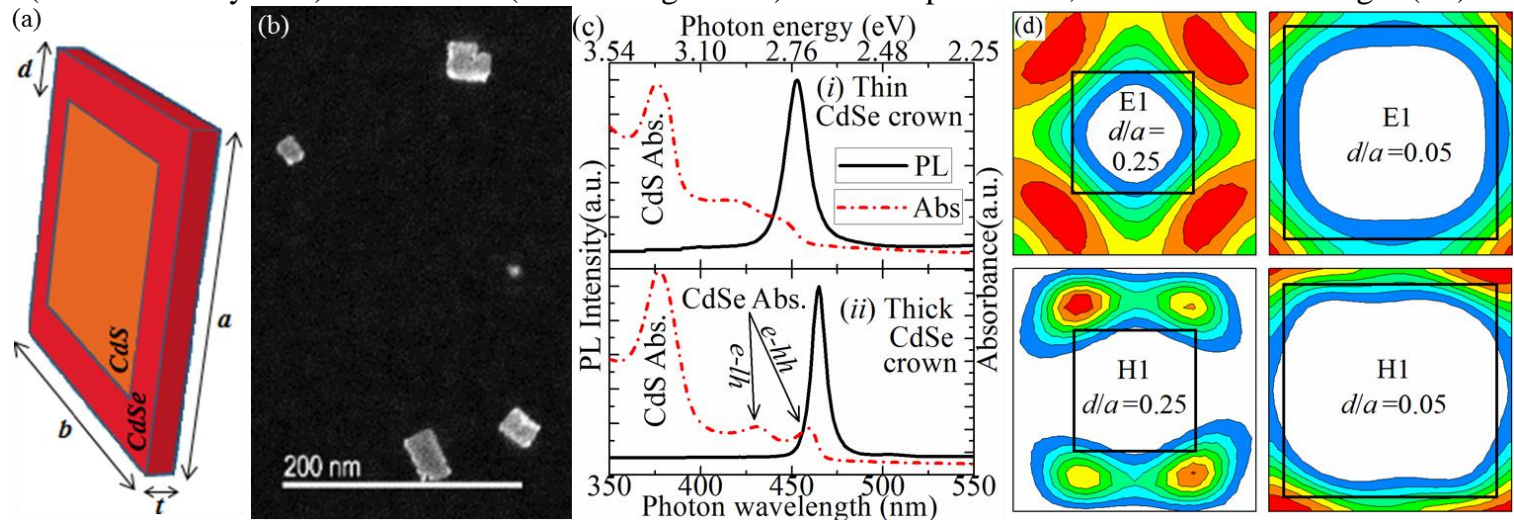

Fig. 1 (a) Schematic of a typical inverted Type-I core/crown CdS/CdSe QR of dimensions $a \times b \times t$ and ring width $d$, (b) Transmission Electron Microscopy (TEM) images of 3ML thick CdS/CdSe QRs (scale: $200 \mathrm{~nm}$ ), (c) PL spectra excited at $350 \mathrm{~nm}$ and absorption spectra for (i) thin and (ii) thick CdSe crown - there is a redshift and fall in linewidth as ring width increases. CdSe and CdS absorption peaks are indicated, (d) Spatial charge density distributions of the first conduction (E1) and first valence (H1) states for thick $(d / a=0.25)$ and thin $(d / a=0.05)$ CdSe crown for square QRs. Warmer (reddish) colors depict higher electron/hole occupation probability over cooler ones (bluish).

We have also studied the 3ML CdS/CdSe QRs theoretically using an effective mass envelope function theory based on the 8-band $\boldsymbol{k} \cdot \boldsymbol{p}$ model [4]. Fig. 1(d) shows the spatial charge density distributions of the first conduction (E1) and first valence (H1) states for thick $(d / a=0.25)$ and thin $(d / a=0.05)$ CdSe crown for square QRs. We can comprehend the extent of quantum confinement, as the photo-generated charge carriers due to ultrafast nonradiative relaxations move to the lowest excited and emissive states in the CdSe crown near the band edge.

$\mathrm{CdS} / \mathrm{CdSe} \mathrm{QRs}$ have already found application as phosphor for color conversion in white light LEDs [3]. They are also potential candidates for magneto-optical device applications (using Aharonov-Bohm effect) [2].

\section{References}

[1] S. Lamarre, É. Rochette, S. Tremblay, C. N. Allen, arXiv:1509.07793v2

[2] I. Fedin and D. V. Talapin, J. Am. Chem. Soc., 138 (31), 9771-9774 (2016)

[3] S. Delikanli, B. Guzelturk, P. Martínez, T. Erdem, Y. Kelestemur, M. Olutas, M. Akgul, H. V. Demir, Adv. Funct. Mater 25, 4282 (2015)

[4] S. Bose, Z. Song, W. J. Fan, and D. H. Zhang, J. Appl. Phys., 119, 143107 (2016) 